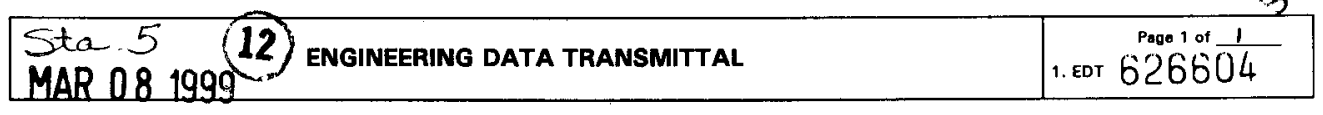

\author{
2. To: (Receiving Organization) \\ DISTRIBUTION \\ 5. Proj./Prog./Dept./DN.: \\ ESPC 291-Z
}

8. Originator Remarks:

A USQ is not required for the test procedure since the test is done off-line at MO-016. A USQ will be issued with the software ECN at the completion of the test.

11. Receiver Remarks: 11A. Design Baseline Document? $\bigcirc$ Yes $O$ No

3. From: (Originating Organization)
PFP Systes Engineering
$\begin{aligned} & \text { 6. Design Authority/Design Agent/Cog. Engr.: } \\ & \text { G. R. Silvan }\end{aligned}$

3. From: (Originating Organization)

6. Design Authority/Des
G. Rilvan

No
6. Design Authory/Design AgentCog. Engr.

4. Related EDT No.:

N/A

7. Purchase Order No::

N/A

9. Equip./Component No::

N/A

10. System/Bidg./Facillity:

93/2721-z

12. Major Asem. Dwg. No.:

N/A

13. Permit/Permit Application No.:

N/A

14. Required Responee Date:

$3 / 10 / 99$

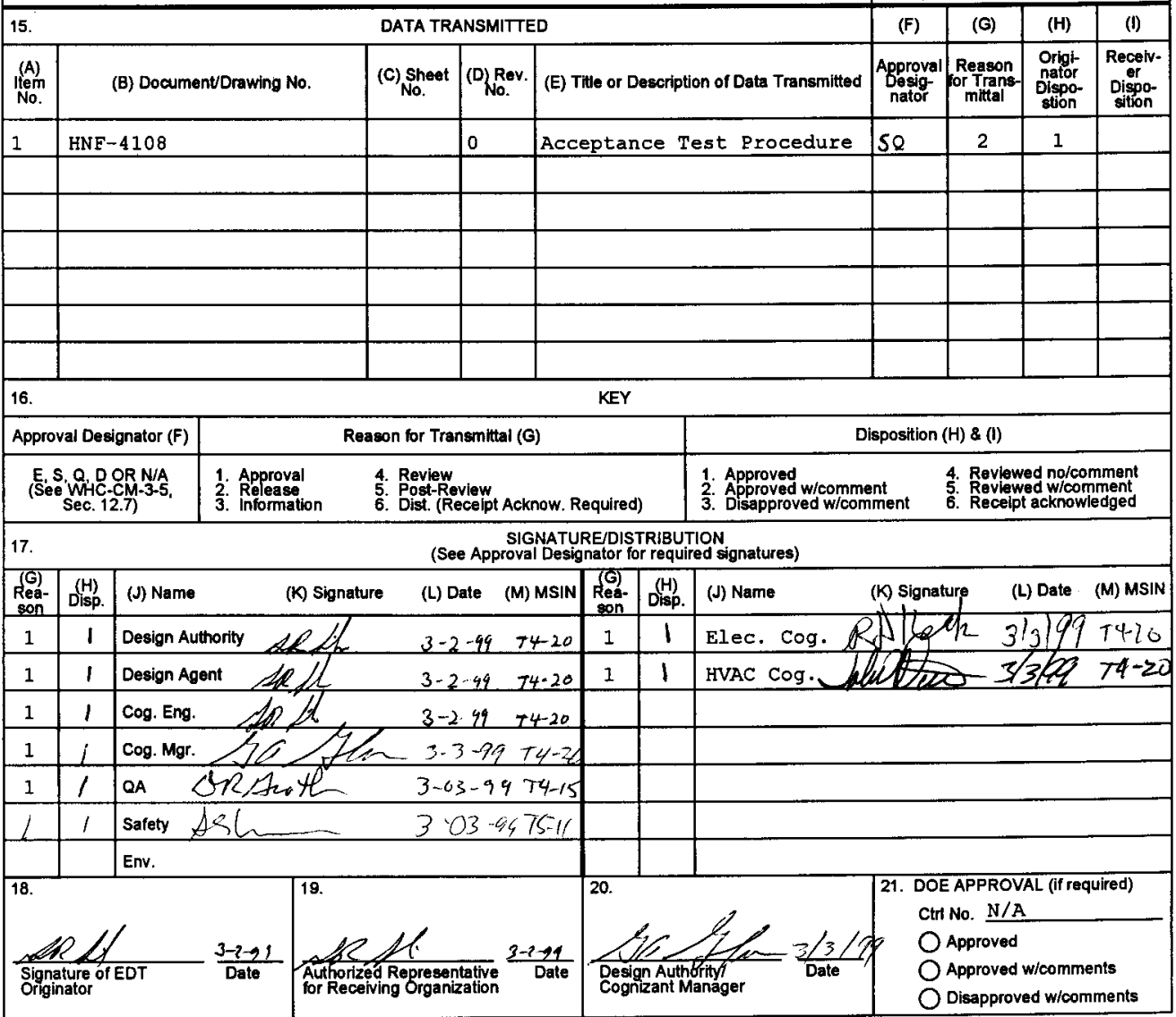


HNF-4108, Rev. 0

\title{
Acceptance Test Procedure MICON Software Exhaust Fan Control Modifications
}

\author{
6. R. Silvan
}

Babcock \& Wilcox Hanford Company, Richland, WA 99352

U.S. Department of Energy Contract DE-AC06-96RL13200

$\begin{array}{lll}\text { EDT/ECN: } & 626604 & \text { UC: } 515 \\ \text { Org Code: } & 15510 & \text { Charge Code: } 103398 \\ \text { B\&R Code: } & \text { EW7002010 } & \text { Total Pages: } 14\end{array}$

Key Words: Generators, Backup Power, Control, Exhaust Fans, MICON

Abstract: This acceptance test verifies the MICON program changes for the new automatic transfer switch ATS-2 alarms, the Closed Loop Cooling isolator status, the CB-3 position alarm, and the alarms for the new emergency fan damper backup air compressor.

MICON is a trademark of MICON Systems Inc.

TRADEMARK DISCLAIMER. Reference herein to any specific commercial product, process, or service by trade name, trademark, manufacturer, or otherwise, does not necessarily const itute or imply its endorsement, recommendation, or favoring by the United states Government or any agency thereof or its contractors or subcontractors.

Printed in the United States of America. To obtain copies of this document, contact: Document Control Services, P.O. Box 950, Mailstop H6-08, Richland wA 99352, Phone (509) 372-2420; Fax (509) 376-4989.

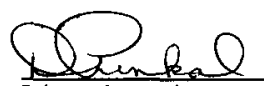

Release Approval

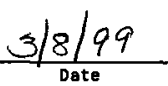

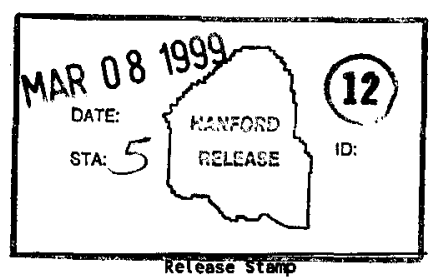

\section{Approved for Public Release}




\section{HNF-4108 Rev. 0}

Acceptance Test Procedure

MICON Software

Exhaust Fan Control

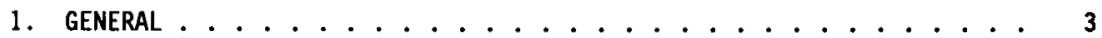

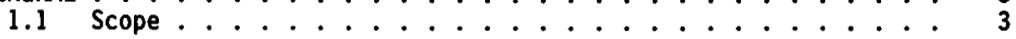

1.2 References ..................... . . . 3

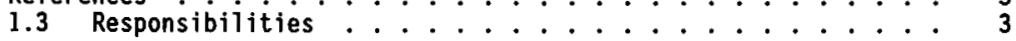

1.4 Software Requirements . . . . . . . . . . . . . . . . . 4

1.5 Test Conditions and Equipment Required ............ 4

1.6 Change Control .................... 4

1.7 Occupational Safety and Health .............. 5

1.8 Exceptions ................... 5

2. ACCEPTANCE TEST ......................... . . . 6

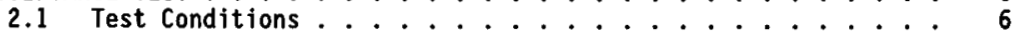

2.1.1 Test Terminology................. 6

2.1.2 Analog Test Box Connections . . . . . . . . . . 7

2.2 Test ATS-2 alarms ................ 8

2.2.1 Digital Test Box Connections............ 8

2.3 Re-test of ATS-2 Start Call (Partial)............ 9

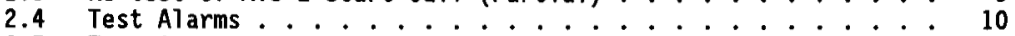

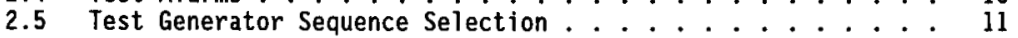

3. EXCEPTIONS TO ACCEPTANCE TEST . . . . . . . . . . . . . . 12

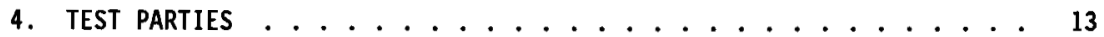

5. TEST APPROVAL . . . . . . . . . . . . . . . . . . . . 14 


\section{HNF-4108 Rev. 0 \\ Acceptance Test Procedure \\ MICON Software \\ Exhaust Fan Control}

\section{GEMERAL}

\subsection{Scope}

This test procedure specifies instructions for acceptance testing of software modifications for alarm changes and additions to the exhaust fan control under Project ESPC (Energy Savings Performance Contract). The software controls the operation of two emergency exhaust fans when there is a power failure.

\subsection{References}

Drawings

400474-101 Rev. A - "RVSS Wiring Diagram 91TE W/CPT NEMA 12 w/Isolation" Engineering Change Notices (ECN)

\section{ESPC-643006}

Prior to final test approval, enter below ECNs written against this ATP.

\subsection{Responsibilities}

\subsubsection{General}

Each company or organization participating in this ATP will designate personnel to assume the responsibilities and duties as defined herein for their respective roles. The designees shall become familiar with this ATP and the systems involved to the extent that they can perform their assigned duties.

1.3.2 Test Director (PFP Electrical Power and Control Cognizant Engineer or MICON Cognizant Engineer)

Coordinates and directs acceptance testing.

Initials each test section as it is completed.

Initiates ECNs to document required changes to the ATP.

Reviews recorded data, discrepancies, and exceptions.

Obtains information or changes necessary to clear or resolve objections during the performance of the test. 
HNF-4108 Rev. 0

Signs Test Approval page when test has been performed.

Obtains required signatures on the ATP Master prior to reproduction and distribution.

1.3.3 WITNESSES (Provided by Participating Organizations. One witness shall be a QC inspector.)

Witness the tests.

Review results of testing.

Assist the Test Director when requested.

\subsection{Software Requirements}

The following software changes shall be verified during this test:

1. Both the ATS-2 Start Call and ATS-2 Emergency Power Bypass inputs shall cause alarms and command the generators to start.

2. The ATS-2 Normal Power Bypass, Lo Damper Air Pressure, and CB-3 Closed inputs shall cause alarms.

3. The Load Isolator Status input shall cause a status change on the generator display.

4. The CB-3 Open/Closed outputs have been disabled.

5. The generator sequence does not reset to 123 whenever the lamp test button is pushed.

1.5 Test Conditions and Equipment Required

Supplied by Test Operator unless otherwise noted.

There will be one analog and one digital test box to simulate inputs to the MICON controller cards in order to test and verify the MICON logic.

\subsection{Change Control}

Technical changes to this test procedure shall be authorized by an ECN. Technical changes shall be signed by the Cognizant Engineer and the QA Engineer or his representative. Record these changes on the exception sheet in Section 3 and section 1.2. Administrative changes, including order of turning on/off inputs or verifications, may be authorized by the Electrical Power and Control or MICON Cognizant Engineer and shall be recorded in black ink on the instruction page. Errors/exceptions in the ATP itself shall NOT be processed as test exceptions. Also, individual test sections may be done in any order at the Test Director's discretion. 
HNF-4108 Rev. 0

1.7 Occupational Safety and Health

In order to accomplish the work in this ATP, it is necessary to have the equipment or circuit energized. Complete testing requires installation and removal of a 24 VDC test box.

\subsection{Exceptions}

Exceptions to the required test results shall be sequentialiy numbered and recorded on the exception forms.

Number each exception sequentially as it occurs and record it on the exception form.

Enter disposition to resolve each exception when such determination is made.

NOTE: Errors/exceptions in the ATP itself shall NOT be processed as test exceptions (see Section 1.6 CHANGE CONTROL). Also, individual test sections may be done in any order at the Test Director's discretion and this practice shall not be considered a test exception. 


$$
\text { HNF-4108 Rev. } 0
$$

\section{ACCEPTANCE TEST}

2.1 Test Conditions

\subsubsection{Test Terminology}

Turn ON Move switch on test box from OFF (Open) position to ON (Closed) Position.

Turn OFF Move switch on test box from ON (Closed) position to OFF (Open) Position.

Verify ON Verify the output LED on the specified point is ON.

Verify OFF Verify the output LED on the specified point is OFF.

Verify

alarm Verify alarm on DCS monitor.

Verify

Indication Verify status or analog indication on DCS monitor.

Verify DCS

Event

Printed Verify event printed on DCS line printer.

Set

analog

Adjust knob on analog test box until desired value is obtained (values can be approximate at the Test Director's discretion) 
HNF-4108 Rev. 0

\subsubsection{Analog Test Box Connections}

Connect the Analog Test Box to the following inputs with all inputs set to their normal values.

\begin{tabular}{|c||l|c|c|}
\hline $\begin{array}{c}\text { Input } \\
\#\end{array}$ & Description & $\begin{array}{c}\text { Slot } \\
\text { No. }\end{array}$ & $\begin{array}{c}\text { Point } \\
\text { No. }\end{array}$ \\
\hline 1 & NP-VF1-3 Voltage & IV & 1 \\
\hline 16 & NP-VF1-3 Frequency & III & 1 \\
\hline 26 & Exhaust Plenum Pressure & II & II \\
\hline 27 & Exhaust Plenum Pressure (redundant) & IV & 15 \\
\hline 28 & Fuel Tank Leve & IV & 14 \\
\hline
\end{tabular}


HNF-4108 Rev. 0

\subsection{Re-test of ATS-2 Start Call (Partial)}

\begin{tabular}{|c|c|c|}
\hline Action & ATS-2 & ATS-2 Bypass \\
\hline Turn ON & $\begin{array}{llr}\text { Gen } 1 \text { Mode in Auto } & (2) \\
\text { Gen } 2 \text { Mode in Auto } & (8) \\
\text { Gen } 3 \text { Mode in Auto } & \text { (14) } \\
\text { ATS-2 Engine Start Cal1 } & \text { (5) } \\
\end{array}$ & $\begin{array}{ll}\text { Gen } 1 \text { Mode in Auto } & (2) \\
\text { Gen } 2 \text { Mode in Auto } & (8) \\
\text { Gen } 3 \text { Mode in Auto } & (14) \\
\text { ATS-2 Emer Pwr Bypass } & \text { (12) } \\
\end{array}$ \\
\hline Verify oN & $\begin{array}{l}\text { Gen } 1 \text { Start } \\
\text { (Slot I Point 4) } \\
\text { Gen } 2 \text { Start } \\
\text { (Slot II Point 4) } \\
\text { Gen } 3 \text { Start } \\
\text { (Slot III Point 4) }\end{array}$ & $\begin{array}{l}\text { Gen 1 Start } \\
\text { (Slot I Point 4) } \\
\text { Gen } 2 \text { Start } \\
\text { (Slot II Point 4) } \\
\text { Gen 3 Start } \\
\text { (Slot III Point 4) }\end{array}$ \\
\hline $\begin{array}{l}\text { Verify alarm } \\
\text { and DCS event } \\
\text { printed }\end{array}$ & ATS-2 Normal Power Fail & ATS-2 Emergency Power Bypass \\
\hline Turn ON & Gen 1 Running & Gen 2 Running \\
\hline $\begin{array}{l}\text { Verify a } 0.2 \\
\text { second pulse } \\
\text { repeating } \\
\text { every } 12 \text { sec }\end{array}$ & $\begin{array}{l}\text { Gen } 1 \text { Sync Aux } \\
\text { (Slot I Point 7) }\end{array}$ & $\begin{array}{l}\text { Gen } 2 \text { Sync Aux } \\
\text { (S1ot II Point } 7 \text { ) }\end{array}$ \\
\hline Turn oN & Gen 1 On Line & Gen 2 On Line \\
\hline Verify OFF & $\begin{array}{l}\text { CB-3 Close } \\
\text { (Slot II Point 11) }\end{array}$ & $\begin{array}{l}\text { CB-3 Close } \\
\text { (Slot II Point 11) }\end{array}$ \\
\hline $\begin{array}{l}\text { Verify pulse } \\
\text { stops }\end{array}$ & $\begin{array}{l}\text { Gen } 1 \text { Sync Aux } \\
\text { (STot I Point 7) }\end{array}$ & $\begin{array}{l}\text { Gen } 2 \text { Sync Aux } \\
\text { (Slot II Point 7) }\end{array}$ \\
\hline Verify on & $\begin{array}{l}\text { Generator on Line } \\
\text { (Slot I Point 17) }\end{array}$ & $\begin{array}{l}\text { Generator On Line } \\
\text { (Slot I Point 17) }\end{array}$ \\
\hline \multicolumn{3}{|l|}{ Wait $30 \mathrm{sec}$} \\
\hline $\begin{array}{l}\text { Verify alarm } \\
\text { and DCS event } \\
\text { printed }\end{array}$ & "ATS-2 failed to transfer" & "ATS-2 failed to transfer" \\
\hline Turn ON & CB-3 Closed & $C B-3$ Closed \\
\hline Turn OFF & Gen I On Line & Gen 2 On Line \\
\hline Verify OFF & $\begin{array}{l}\text { CB-3 Open } \\
\text { (Slot II Point 12) }\end{array}$ & $\begin{array}{l}\text { CB-3 Open } \\
\text { (S1ot II Point 12) }\end{array}$ \\
\hline $\begin{array}{l}\text { Test } \\
\text { Successful }\end{array}$ & & \\
\hline
\end{tabular}


HNF-4108 Rev. 0

\subsection{Test Alarms}

\begin{tabular}{||l|l||}
\hline Action & Alarm \\
\hline Turn ON & ATS-2 Norma1 Power Bypass (18) \\
\hline $\begin{array}{l}\text { Verify alarm and DCS event } \\
\text { printed }\end{array}$ & ATS-2 Normal Power Bypass \\
\hline Turn OFF & ATS-2 Normal Power Bypass (18) \\
\hline Verify alarm cleared & ATS-2 Normal Power Bypass \\
\hline Turn ON & Damper Air Low Pressure (19) \\
\hline Verify alarm cleared & Damper Air Low Pressure \\
\hline Turn OFF & Damper Air Low Pressure (19) \\
\hline $\begin{array}{l}\text { Verify alarm and DCS event } \\
\text { printed }\end{array}$ & Damper Air Low Pressure \\
\hline Turn ON & CB-3 Closed (11) \\
\hline Verify alarm cleared & CB-3 Open No Emergency Power to Fans \\
\hline Turn OFF & CB-3 Closed (11) \\
\hline $\begin{array}{l}\text { Verify alarm and DCS event } \\
\text { printed }\end{array}$ & CB-3 Open No Emergency Power to Fans \\
\hline Turn ON & CLC Load Isolator Status (17) \\
\hline \begin{tabular}{l} 
Verify Closed \\
\hline Turn OFF
\end{tabular} & CLC isolation contact display \\
\hline Verify Open & CLC isolation contact display \\
\hline $\begin{array}{l}\text { Test } \\
\text { Successful }\end{array}$ & CLCad Isolator Status (17) \\
\hline
\end{tabular}


HNF-4108 Rev. 0

\subsection{Test Generator Sequence Selection}

\begin{tabular}{|c|c|}
\hline Action & Generator Sequence \\
\hline $\begin{array}{l}\text { Turn oN for two seconds, } \\
\text { then turn OFF }\end{array}$ & 123 Sequence $(20)$ \\
\hline Verify on & 123 Sequence Light (Slot I, Point 3) \\
\hline Verify OFF & $\begin{array}{l}231 \text { Sequence Light (Slot II, Point 3) } \\
312 \text { Sequence Light (Slot III, Point 3) }\end{array}$ \\
\hline $\begin{array}{l}\text { Turn for five seconds, } \\
\text { then turn OFF }\end{array}$ & Lamp Test (23) \\
\hline Verify ON & 123 Sequence Light (S1ot I, Point 3) \\
\hline Verify OFF & $\begin{array}{l}231 \text { Sequence Light (Slot II, Point 3) } \\
312 \text { Sequence Light (Slot III, Point 3) }\end{array}$ \\
\hline $\begin{array}{l}\text { Turn on for two seconds, } \\
\text { then turn OFF }\end{array}$ & 231 Sequence (21) \\
\hline Verify oN & 231 Sequence Light (Slot II, Point 3) \\
\hline Verify OFF & $\begin{array}{l}123 \text { Sequence Light (S1ot I, Point 3) } \\
312 \text { Sequence Light (S1ot III, Point 3) }\end{array}$ \\
\hline $\begin{array}{l}\text { Turn oN for five seconds, } \\
\text { then trun OFF }\end{array}$ & Lamp Test (23) \\
\hline Verify on & 231 Sequence Light (Slot II, Point 3) \\
\hline Verify OFF & $\begin{array}{l}123 \text { Sequence Light (Slot I, Point 3) } \\
312 \text { Sequence Light (Slot III, Point 3) }\end{array}$ \\
\hline $\begin{array}{l}\text { Turn ON for two seconds, } \\
\text { then turn OFF }\end{array}$ & 312 Sequence (22) \\
\hline Verify oN & 312 Sequence Light (S1ot III, Point 3) \\
\hline Verify OFF & $\begin{array}{l}123 \text { Sequence Light (S1ot I, Point 3) } \\
231 \text { Sequence Light (S1ot II, Point 3) }\end{array}$ \\
\hline $\begin{array}{l}\text { Turn oN for five seconds, } \\
\text { then trun OFF }\end{array}$ & Lamp Test (23) \\
\hline Verify oN & 312 Sequence Light (S1ot III, Point 3) \\
\hline Verify OFF & $\begin{array}{l}123 \text { Sequence Light (Slot I, Point 3) } \\
231 \text { Sequence Light (Slot II, Point 3) }\end{array}$ \\
\hline $\begin{array}{l}\text { Test } \\
\text { Successful }\end{array}$ & \\
\hline
\end{tabular}


HNF-4108 Rev. 0

3. EXCEPTIONS TO ACCEPTANCE TEST

\begin{tabular}{|c|c|c|c|c|}
\hline ITEM & STEP & PAGE & DESCRIPTION & DISPOSITION \\
\hline & & & & \\
\hline & & & & \\
\hline & & & & \\
\hline & & & & \\
\hline & & & & \\
\hline & & & & \\
\hline & & & & \\
\hline & & & & \\
\hline & & & & \\
\hline & & & & \\
\hline & & & & \\
\hline & & & & \\
\hline & & & & \\
\hline & & & & \\
\hline & & & & \\
\hline & & & & \\
\hline & & & & \\
\hline
\end{tabular}




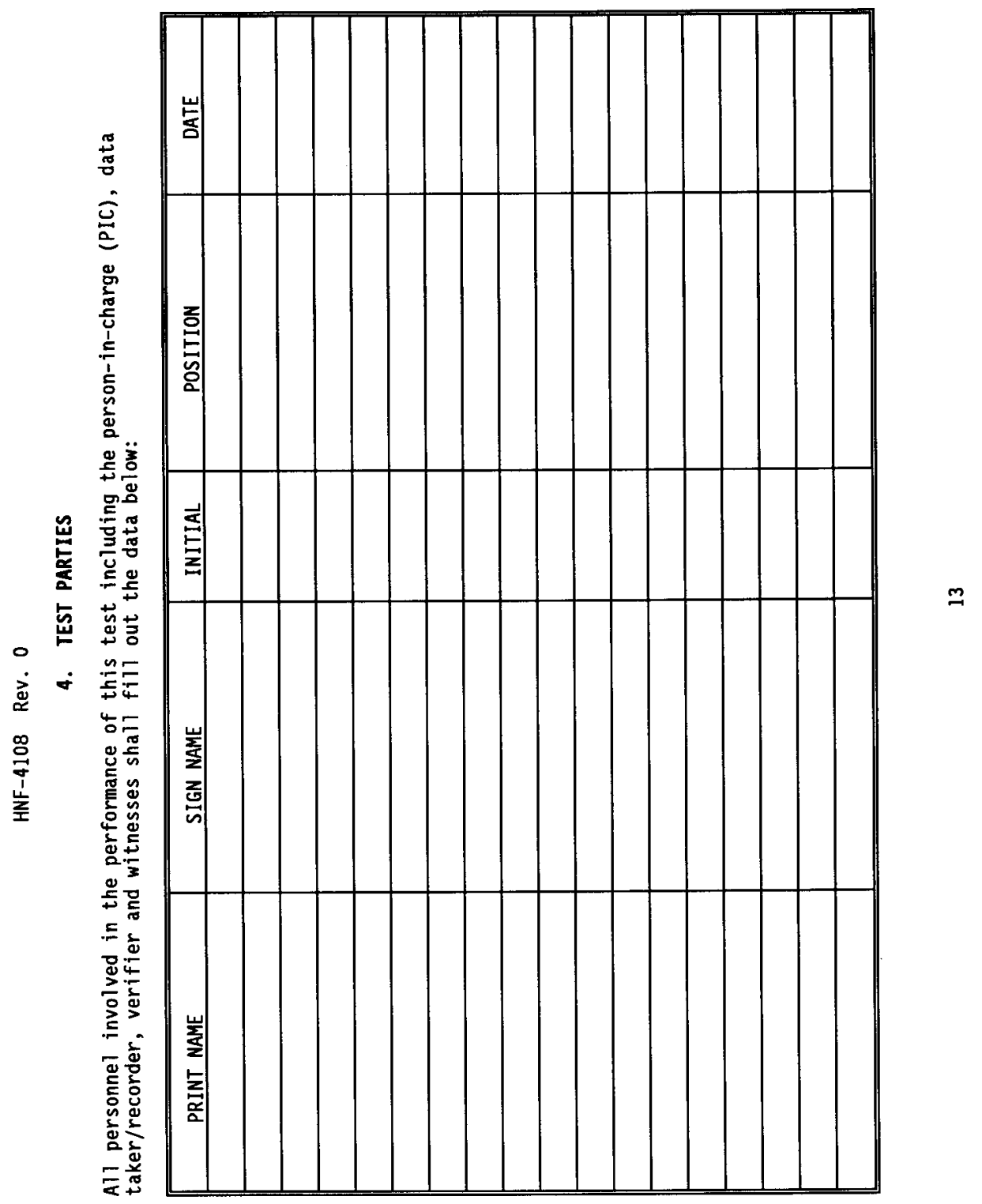


HNF-4108 Rev. 0

5. TEST APPROVAL

Without

exceptions
With exceptions

resolved

With exceptions

outstanding

\begin{tabular}{lc}
\hline PFP Electrical Cognizant Engineer & Date \\
\hline MICON Cognizant Engineer & Date \\
\hline QC Inspector & Date
\end{tabular}




\section{DISTRIBUTION SHEET}

To

Information Resource ManagementPF

Project Títle/Work Order

ESPC 291-Z

Name

RD Keck

GL Rippy

GR Silvan

Central Files

DOE-RL Reading Room
From

PEP Eacility system Engineering

\begin{tabular}{|l}
\hline \\
\hline \\
\hline
\end{tabular}

DoE RI Reading Room

Page 1 of 1

Date $3 / 1 / 99$

EDT No. 626604

ECN No. N/A

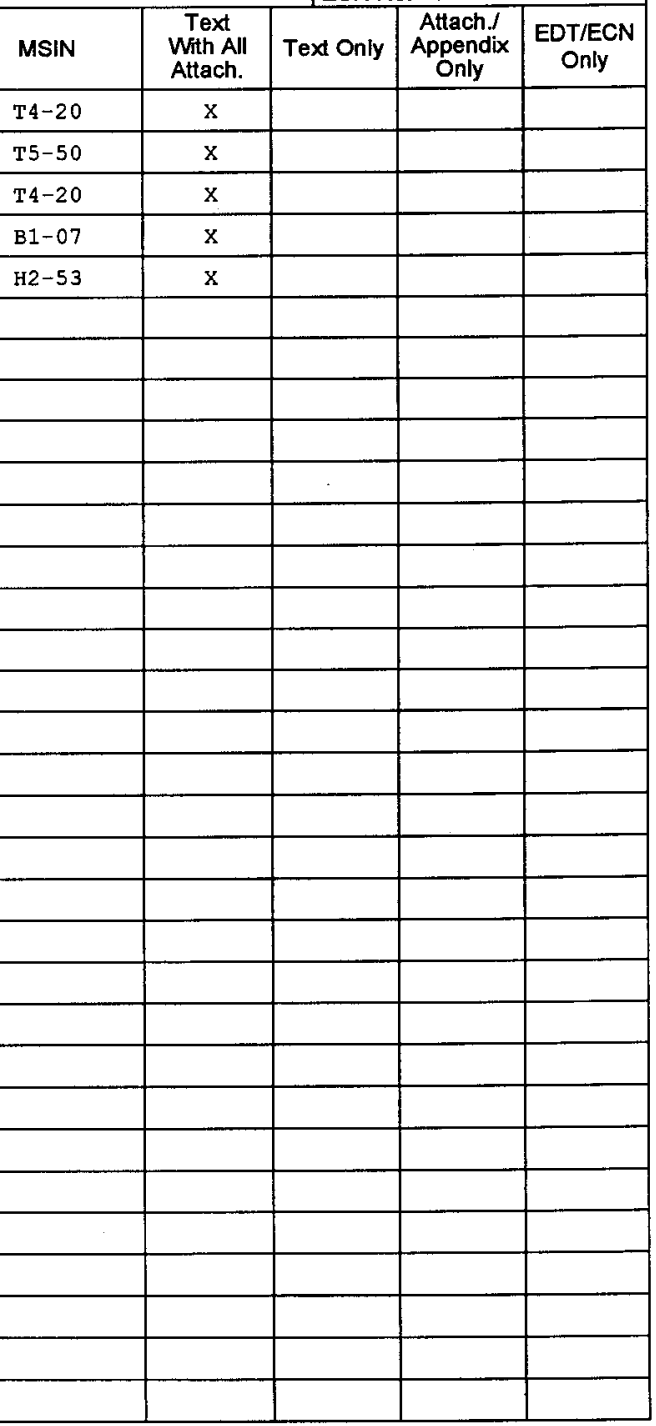

